\title{
Internet Rescue Robots for Disaster Management
}

\author{
Punith Kumar M B \\ Associate Professor, Department of ECE, PES College of Engineering Mandya, Karnataka, India \\ Email:punithpes@gmail.com
}

\section{Sumanth S}

Assistant Professor, Department of ECE, PES College of Engineering Mandya, Karnataka, India

Email: sumanthsmandya@gmail.com

\section{Manikant Amaresh Savadatti}

Dept, of ECE, PESCE, Mandya, India

Email: manisavadatti@gmail.com

Received: 12 February 2021; Accepted: 28 March 2021; Published: 08 April 2021

\begin{abstract}
The prototype development of Rescue Robot can help search and rescue teams with their difficult and lifesaving tasks. Unmanned Aerial vehicles (UAVs) and Unmanned Ground vehicles (UGVs) are viewed as two separate entities. However, upon closer examination, we can classify them into a closely-coupled system where both are just seen as separable parts of the body of one robotic entity, whose body parts can separate temporarily and acquire together again later. In this prototype, a mobile robot UGV can be used as transport and landing station for a lightweight quadcopter UAV. UAV has separable long-range vision system which provides the top view of environment for the UGV, separable images are stitched and transformed into maps and utilized for rescue operations. This rescue robot contains a combination of UGV and UAV. It is equipped with a strong manipulator arm, used for debris removal, shoring operations, and delivering medicines/ food on very rough terrain. It has light detection and ranging (lidar) sensors, cameras, enabling it to look for victims and for mapping semi-destroyed buildings. The UGV can send visual feedback to the operator at a remote location. The UAV drone has a camera for an aerial view and mapping of the place. Working in conjunction with one another and thus the human search and rescue workers, these robotic assets form a strong team, increasing the effectiveness of search and rescue operations. This project aims at the realization of a new generation of search and rescue robot which can work in semi-autonomous and wireless modes and can be used in harsh physical environments of disaster regions to hold out the given tasks more effectively by the utilization of advanced and economic sensors. the problem domain of earthquake disasters and search and rescue processes is clarified with introducing an overview of this project. Disasters unsettle the economic and social stability of society. Shortage of skilled rescue work forces, also because the risks involved in search and rescue operations, are getting foremost problems during an emergency situation. In this paper rescue robot were designed, implemented in a real time environment and analyzed the result.
\end{abstract}

Index Terms: Unmanned Ground Vehicle, Unmanned Aerial Vehicle, Internet Robot, WIFI Robot, Rescue Robot

\section{Introduction}

Robotics is an important field in the era of automation. Unlike a person, a computer-controlled robot can work with speed and accuracy without feeling exhausted. A robot can also perform pre-assigned tasks during a hazardous environment, reducing the risks and threats of the citizenry. For example, a rescue robot can rapidly detect, locate, and neutralize all sorts of threats, including chemical and biological agents, and impossible terrain or unusable routes or roads.

The Main objective of this work is to be able to develop robots that can go inside the site instead of humans, several area of robotics need to be addressed and integrated inside a common robotic platform. To facilitate such integrations. Here they developed a highly maneuverable mobile robot, which contains software and hardware architecture keeping in mind that collaboration between research and development groups of different interests. In this paper, the problem domain of earthquake disasters and search and rescue processes is clarified with introducing an overview of this project

UGV and UAV are being developed for both civilian and military use to perform dangerous activities. They are very effective in a large number of circumstances where the use of human labor is too expensive, the task is risky, or it 
is impractical for human capability. There are two general classes of unmanned mobile vehicles: Teleoperated, just like the one described within the present work and Autonomous. A Teleoperated or unmanned guided vehicle. A UGV may be a vehicle that's controlled by a person's operator at a foreign location via a communications link.

Unmanned aerial vehicles (UAVs) can provide real-time visual feedback and data within the aftermath of an earthquake or hurricane or any natural disaster. They will also become an eye fixed within the sky to locate a lost person within the mountain for instance. When a disaster or incident threatens lives and livelihoods, emergency responders need information and real-time imagery so as to make better decisions and save time. UAVs can provide awareness over a huge area quickly, reducing the time and thus the number of searchers required to locate and rescue an injured or lost person, greatly reducing the value and risks of search and rescue missions. The chances for helping ensure public safety are endless .UAVs still have lots of benefits as compared to UGV robots: they have a large area to cover which usually has much fewer obstacles, can often have higher instantaneous velocities as compared to ground vehicles. Most significantly, they will observe a larger area through their sensors.

On the opposite hand, UGV robots also have numerous advantages too: they will carry larger loads than comparable UAVs, and thus they will carry much larger power sources. However, as long as they have to remain in touch with the bottom, they're much more vulnerable to obstacles, harsh terrain, also as occlusions, and other sensing limitations. The combination of those two sorts of robots, though, and their conceptualization as a single heterogeneous hybrid system with on-the-fly separable and combinable body parts can create highly beneficial scenarios

\section{Literature Review}

In the past, related research has taken place within the area of multi-agent systems such as UAV-UGV cooperation [1,2,3] and multi-robots[4,5] like multi-UAVs [6,7] and multi- UGVs[8,9,10]. UAVs and UGVs have several advantages and disadvantages over each other in terms of movement, weight carrying capacity, and perception abilities and past work [2,3] focus on combining UAVs' and UGVs' advantages together to create a multi-robot system that will be implemented to try to a specific task as in [1,2,7]. Research in the multi-robot system has been done because it has many advantages and applications in many fields like the military, surveillance, etc. UAVs and UGVs can work simultaneously used for exploring unknown areas When multiple robots are present and to be controlled simultaneously, the system requires careful attention on how each robot behaves so as to avoid any collision or any unexpected scene [11]. But past work tends to use one sort of mobile robots, either UAV [6,7,9] or UGV [3,10] to fulfill the task. In this paper, it consists of UGV with a UAV that explores and maps the unknown indoor environment using motion capture to get the situation of the UAV and a camera mounted on the UAV to take an aerial view. There has been an identical work of indoor environment mapping [8] using UGVs and a sick laser. In our work, the UAV provides a clear aerial view of the unknown environment and therefore the main system installed on UGV creates a Lidar map cloud. There are three types of moving mechanisms for this kind of robots in general: wheel type [12], track type [13], and walking type mechanism [14]. The wheel-type mechanisms are inferior to track-types when they are to move on rough terrain. Walking robots have good dexterity on rugged terrain, but its complex structures usually make its control difficult. In that sense, the track mechanism has good mobility under rough ground conditions.Hence this work consists of tracked mechanism .

\section{Features and Advantages}

1. Our robot can move at a speed of $32 \mathrm{~cm} / \mathrm{sec}$.

2. As a Lidar sensor for distance mapping. It can measure up to 2 meters.

3. Our robot is a drone for an aerial view.

4. But recent robots don't have a drone and rescue robots in one package, but our robots do both.

5. All robots use radio frequency to control. But our robot uses the Internet.

6. Our robot is a holder for a water bottle which is necessary for survivors.

7. It also has two cameras, one for the crawler and another one for the drone.

8. Since it has a camera attached to the robotic arm it can capture 360 areas since the arm has 360 freedoms. The Crawlers camera is equipped with LEDs for nighttime.

9. It's a drone that can be controlled both by $\mathrm{Wi}-\mathrm{Fi}$ and radio frequency.

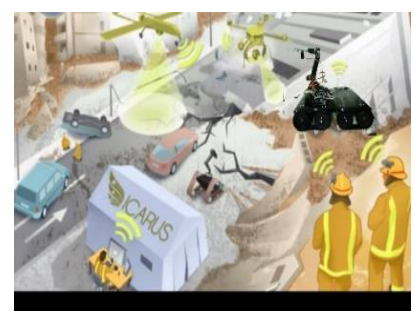

Fig.1. Controller Operating Robots 


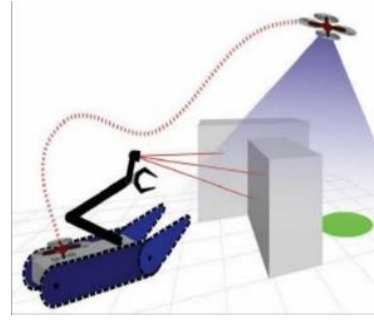

Fig.2. UGV and UAV Combination

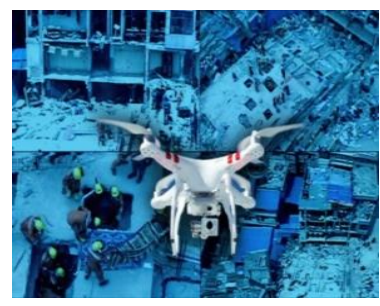

Fig.3.Drone Mapping

Human soldiers are hard-working, but they are still humans, they get tired and make decisions that can risk the whole team. Rescue robots run continuously for a long time and the controller can sit in the base and make better decisions as shown in Fig.1. The other problem is a human can't analyze the situation of the destroyed place quickly but aerial rescue robots can fly and analyze the place very quickly. A human can only get the data to the line of vision. If we use rescue drones we can get the top view and other aerial views which is not possible for humans. These images help the rescue operators to get a better view of the problems and help them plan for rescuing operations as shown in Fig.2. Humans can't reach places that are very small. But rescue robots are small and can be used in such places and humans cannot enter partially collapsed buildings because they will be in fear if the building may collapse hence Internet rescue bots can be engaged in such places without any fear. We can combine the drone-mapped images, stitch them and form one single large map as shown in Fig.3.

\section{Methodology}

We have designed a rubber-tracked wheel mechanism where 2 wheels are connected by a thick rubber belt. There are a pair of rubber belt-track wheel mechanisms on either side of the vehicle. The main 4 wheels are connected to the chassis of the vehicle by $12 \mathrm{v}$ DC motors and the other 4 are dummy wheels used for support as shown in Fig.4. 12 Volt, 2 A (Maximum load current) 4 D.C. motors are used to drive the pair of tracked belt wheels on either side of the chassis The motors are connected to the wheel shaft by a 1:15 reduced gear ratio to scale back the speed and extend the torque of every motor. Each motor can rotate clockwise or anticlockwise in a direction. The chassis of the vehicle is made up of high-strength metal which makes the vehicle strong. The diagonally placed motor can provide the necessary torque to turn the vehicle at a given angle. The vehicle can make 180 degrees turn with almost zero displacements of its center of gravity. The UGV is equipped with a strong manipulator arm shown in Fig.4. Each joint of the robotic arm contains 360 Servo motors with $4 \mathrm{~kg}$-cm torque. Since 360 motors are used each joint of the robotic arm has 360 freedoms of rotation. The robotic arm is built of high-strength aluminum which is strong and light. And the servos used are made up of metal gears that will not wear and tear easily. A lidar sensor is used to form a point cloud which can be used for distance mapping shown in Fig.6. Lidar sensor has a range of around 2 meters. Along with a lidar sensor, we have a Wi-Fi camera mounted at the tip of the robotic arm which can stream live video directly to the smartphone. For night vision we have an led strip that acts as a searchlight during the night. The best thing about this configuration is the camera is mounted on the robotic ARM, and the robot arm has 360 freedoms so it can capture videos and map its 360-surrounding area. It also has an ultrasonic sensor that is used for obstacle detection shown in Fig.6. UGV is controlled by the internet hence it connects to the internet and we can send commands to it from any part of the world. The next major part of our Rescue robot is a drone which is placed on the back of the UGV as seen in Fig.5. The drone contains a Wi-Fi camera that can be used for aerial monitoring of the area. Hence, this rescue robot contains a combination of both UGV and UAV.

The high torque motors, high-strength metal chassi, strong manipulator arm, lidar sensor, a Wi-Fi camera, UAV all these can be used in rescue operation. This robot can be sent into the disaster place like earthquake .A person would be controlling the robotic crawler and drone via internet through a smart phone ,he would be receiving the live video feed from the robotic crawler and also from the drone. After sending the robot to the disaster place the controller can use cameras ,lidar sensor, ultrasonic sensor to scan the environment, and help rescue team in analyzing the disaster place easily. 


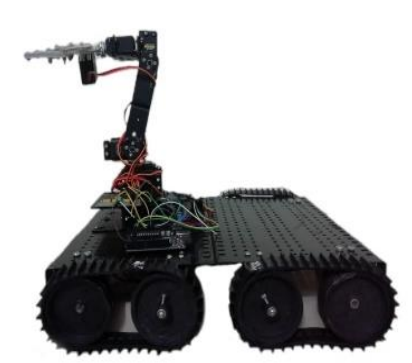

Fig.4.UGV Side View

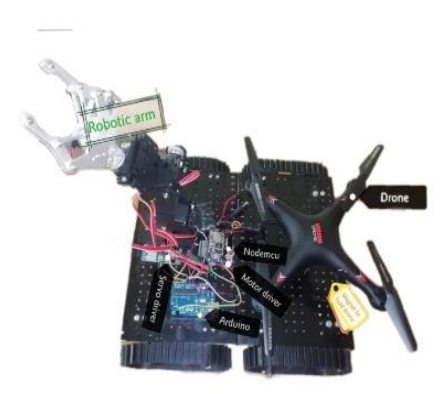

Fig.5.UGV Top View

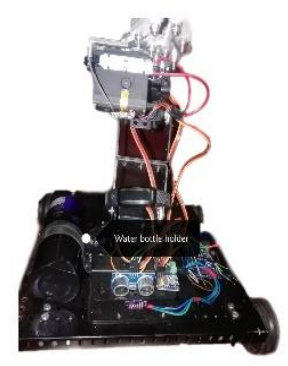

Fig.6. UGV Front View

\section{Hardware}

Below all the components used in building the robot are listed.This list would help other researchers who are interested in advancing the current experiment.

Motors: Permanent-magnet direct-current (PMDC). These motors as shown in Fig.7. They are easy to use because both their direction and speed can be controlled, the direction of polarity, and speed by voltage. DC Motors are mostly used in robotics as shown in figure 5 because of their small size and more energy output, they are best for driving the mechanical assemblies.

1. 150RPM $12 \mathrm{~V}$ DC motors with Gearbox

2. $6 \mathrm{~mm}$ shaft diameter with internal hole

3. $125 \mathrm{gm}$ weight

4. Stall Torque $=1 \mathrm{kgcm}$ torque

5. No-load current $=60 \mathrm{~mA}(\mathrm{Max})$, Load current $=300 \mathrm{~mA}$

Motor Driver: L298N Module is a motor driver module for driving DC and Stepper Motors. This module has an L298 motor driver IC and a 78M05 5V regulator. L298N Module can control up to four DC motors, or just two DC motors with directional and speed control shown in Fig.8.

1. Driver Chip: Double H Bridge L298N

2. Motor Supply Voltage (Maximum): $46 \mathrm{~V}$

3. Motor Supply Current (Maximum): 2A

4. Driver Voltage: $5-35 \mathrm{~V}$

5. Logical Current:0-36Ma 
Robot ARM: It is a programmable mechanical ARM as shown in Fig.9., which functions the same as that of a human ARM. The links of a robotic ARM are connected using servo motors allowing either translational or rotational motion. The robotic ARM has three parts, namely base, elbow, and gripper. Each part has one degree of freedom which means it will have 4 movements. Servo Motor will be used to move the robot ARM and will be located at every joint of the robot ARM.

Fig.7. DC Motor
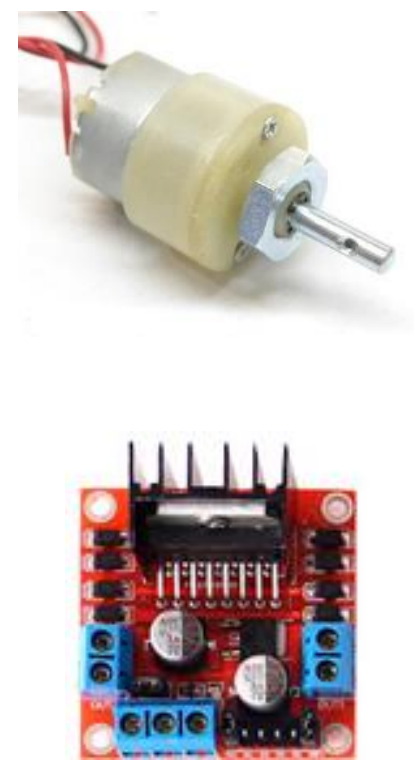

Fig.8. Motor Driver

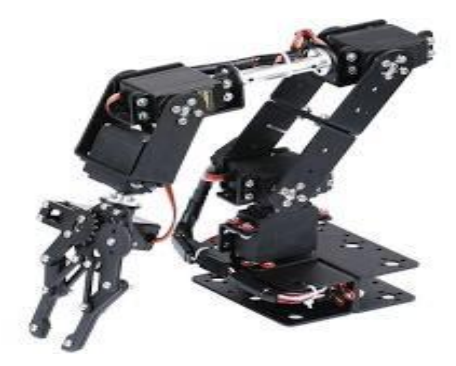

Fig.9. Robotic ARM

NodeMCU: NodeMCU is an open-source development board and firmware based on the widely used ESP8266 12E Wi-Fi module as shown in Fig.10. It allows you to program the ESP8266 Wi-Fi module with powerful LUA programming language or Arduino IDE. Arduino-like hardware I/O, USB-TTL included, it has 10 GPIOs D0-D10 with PWM functionality, IIC, and SPI communication, 1-Wire, and ADC at A0, etc. it is an all in one board. It is an Eventdriven API for network applications with PCB antenna.

Arduino UNO: The Arduino UNO as a microcontroller ATmega328P as shown in Fig.11. Arduino is an opensource platform and its simple design and using ability makes it ideal for hobbyists and professionals. The Arduino UNO has 14 digital I/o pins (6 can be used as PWM outputs), 6 analog input pins, a $16 \mathrm{MHz}$ crystal oscillator, a USB connection port, a dc power jack, an ICSP header, and also a reset button

Battery: Battery pack Capacity: 2000mah, Battery Type: Rechargeable Li-on battery. Input Voltage:12.6V,Output voltage: 11.1 - 12.6v DC , Output Current: About 1-3A, Energy - Nominal Voltage: 11.1V - Output, Voltage Range: 11.1v - 12.6 by test conditions

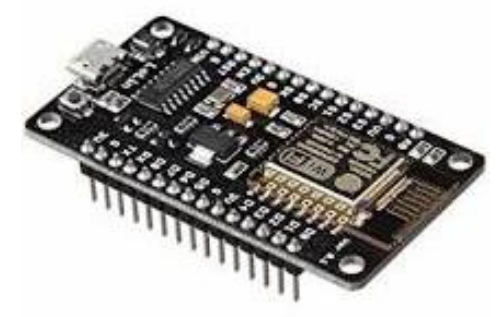

Fig.10. Nodemcu 


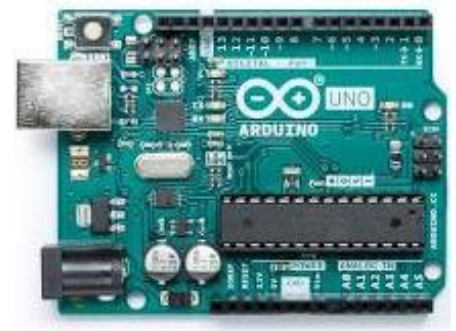

Fig.11. Arduino UNO

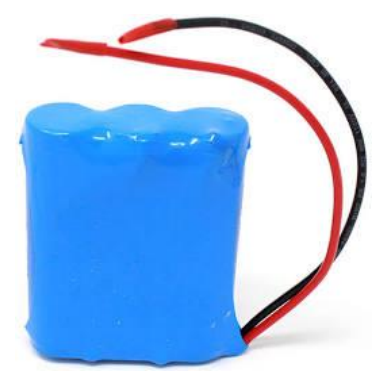

Fig.12. 12v Battery

Wi-Fi FPV Camera: These cameras as shown in Fig.13. Have an integrated Wi-Fi chip through which they stream the live video feed to their mobile phone. It weighs around $200 \mathrm{gm}$. Its dimension is $21 \times 16 \times 7 \mathrm{~cm}$. We can download an app from the play store and the camera will connect to mobile Wi-Fi and streams it to the mobile app. Through which you can record video and capture images

LIDAR: The VL53L0X is a new generation Time-of-Flight (ToF) laser-ranging module housed in the smallest package on the market today, providing accurate distance measurement whatever the target reflectances unlike conventional technologies. It can measure absolute distances up to $2 \mathrm{~m}$, setting a new benchmark in ranging performance levels, opening the door to various new applications as shown in Fig. 14.

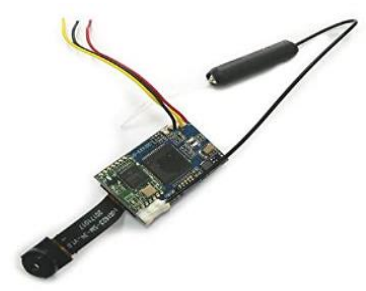

Fig.13. Wifi Camera

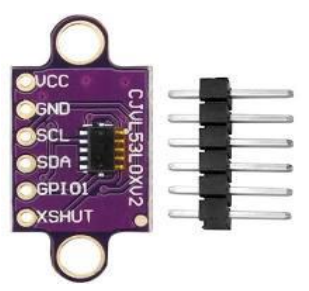

Fig.14. Lidar Sensor

\section{Block Diagram}

Fig.18. the following diagram represents the working block diagram of the Internet Rescue Robot. Let me start from the controller's point of view. A controller would have 2 smartphones, one smartphone would be used as a controller and the other would be used to receive the live video stream. Let's start with a controller, this smartphone would connect to the Blynk server via the blynk app and sends commands to the Wi-Fi module. There are two main microcontrollers in the UGV, one is the Arduino UNO and the other is the Nodemcu Wi-Fi module, both the microcontrollers will talk to each other via serial communication. Nodemcu receives the commands from the smartphone and sends the commands to the Arduino via serial communication. The UGV motion mechanism is controlled by Nodemcu, while the Arduino UNO controls the robotic arm, Lidar, Ultrasonic sensor, and Wi-Fi camera 
with a searchlight. Another smartphone can be used to connect to the Wi-Fi cameras and receive the live video feed. UAV drones can be controlled using a Wi-Fi-enabled smartphone or an RF controller. In the operator location, the smartphone receiver receives the analog video signal, sent by the UGV AND UAV. The operator on seeing the live video feedback from the remote UGV and UAV sends basic navigational instructions (left, right, forward, backward, stop) for proper navigation.

\section{Controller App Overview}

The Fig15.and Fig16. show the overview of a blynk app that can be used to control UGV, Fig.17. shows the overview of the XSW app which can be used to control the UAV. Blynk app consists of sliders, joysticks for controlling the UGV. As shown in Fig.15 the first tab of the blynk app contains a slider for controlling the speed of the UGV. We can move the slider to control the speed with which the UGV moves. The joystick shown in Fig.16. can be used to control the direction of the UGV. We can move the UGV front, back, right, left and the vehicle can make 180 degrees turn with almost zero displacements of its center of gravity. The second image Fig.17. shows four different joysticks that can be used to control each joint of the robotic arm. The joystick can control the 360-degree movement of each joint of the robotic arm. The third image Fig.17. shows the XSW app which can be used to control the UAV as well as receive the live video feed from the camera at a time. These two apps can be used to control the UGV, Robotic arm and the UAV.

This experimentation would help in creating a best rescue robot .

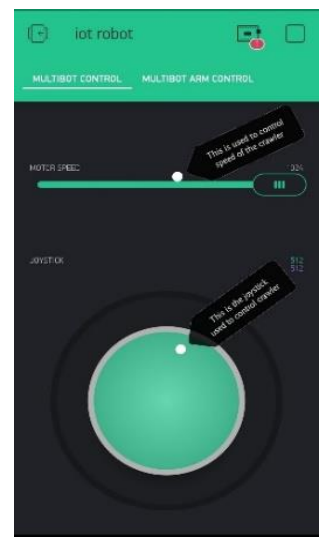

Fig. 15. UGV Controller

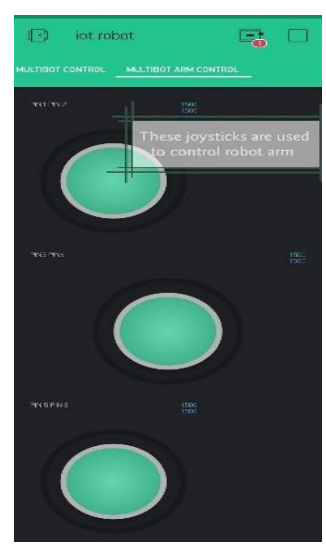

Fig.16. Robotic ARM 


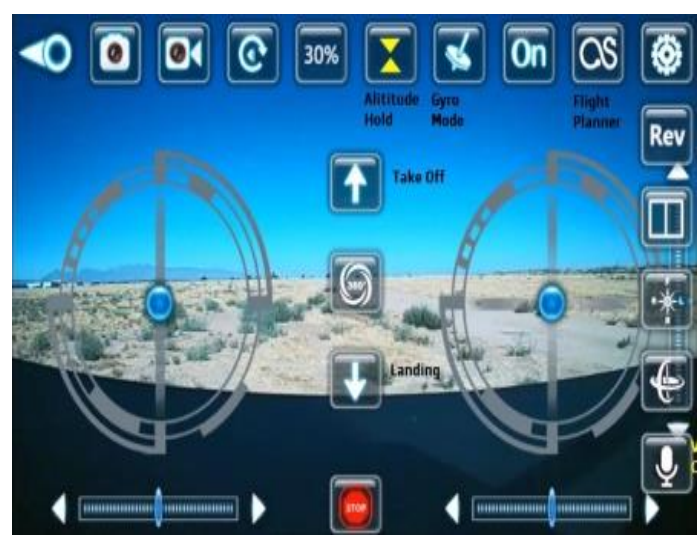

Fig.17. XSW App

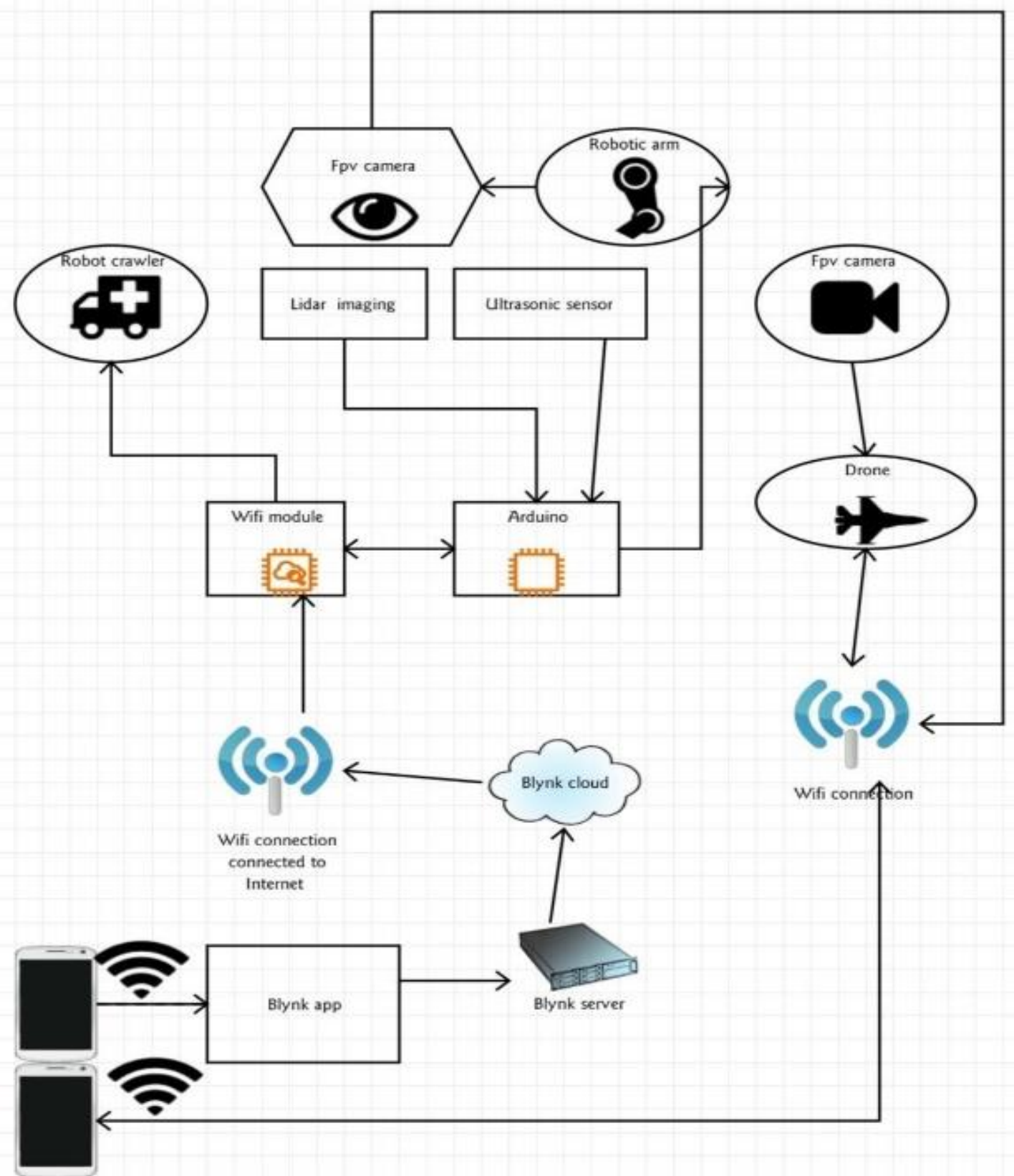

Fig.18. Block Diagram 


\section{Conclusion and Results}

In our present work, we have successfully developed a prototype UGV and UAV capable of traveling through difficult terrains even without daylight. This helps us to achieve our objective of searching for humans even during the night time.The major difficulty faced during experimentation is the low ground clearance of the vehicle. Wheels with bigger diameters may solve this problem. The Wi-Fi camera output is also affected by external noise. The UGV needs more torque for rough terrains which could be easily solved by replacing dummy wheels with high torque motors. The lidar can measure a distance of about 1.5 meters with high accuracy. This will help in creating a $3 \mathrm{~d}$ cloud map of surrounding place from a far distance. The camera mounted on UGV and UAV can stream the video and live images of the locations which can be stitched together and this would create a photo graphic image of the surrounding area

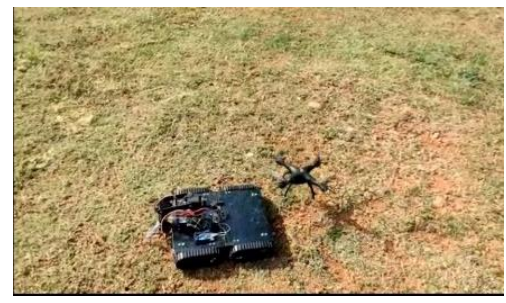

Fig.19. UGV and UAV

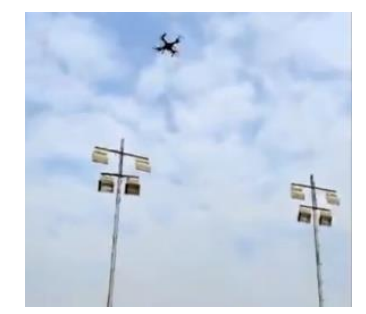

Fig.20. UAV Demo

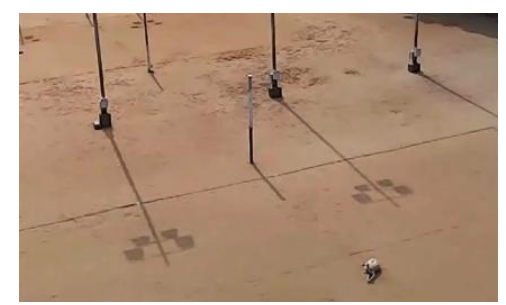

Fig.21.UAV Live Footage

Fig.19. shows the UGV and UAV in action. The UGV is imaging the ground and the UAV is lifting the UGV back for imaging the aerial view.Fig.20. shows the UAV in action flying and sending the live video feedback to the smartphone.Fig.21. is the actual live footage streamed by the UAV. The results we intended to obtain from the UAV is achieved. The Wireless Wi-Fi control and the RF control mechanism of the UAV are working as expected. The working of UAV, lifting of the UAV from the UG back, and live footage sample taken from the drone is shown in the above images

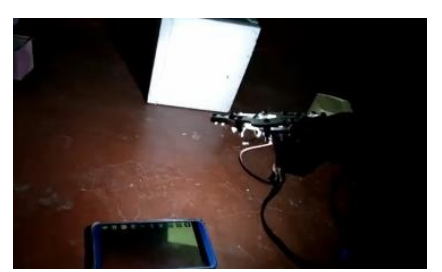

Fig.22.UGV Night Search 


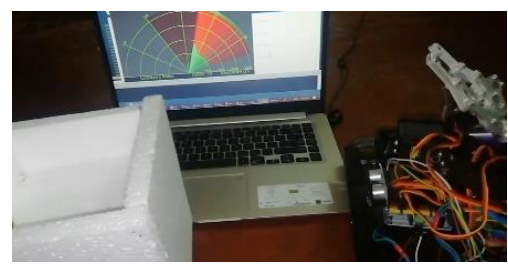

Fig.23. UGV Radar system

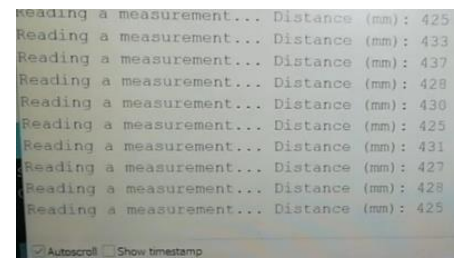

Fig.24. UGV Lidar output

Fig. 22. shows the rescue robot prototype working at night, it shows the results of the searchlight and camera mounted on the robotic arm.Fig.22. shows the rescue robot searching for humans at night time with its searchlight enabled, and also it shows the live video streaming of the camera mounted on the camera. We can see a smart phone streaming live video from the camera mounted on the arm which can be used to view the disaster place .Fig. 23. shows the results of the ultrasonic sensor, the screen of the laptop shows the radar results in visual form. If the object is detected by the ultrasonic sensor, the radar shows a red wave of colors. The closer the object gets the larger the red wave would spread in the radar system. This would help to detect obstacles for the UGV.Hence the robot can avoid hitting obstacles or the debris Fig. 24 shows the output of the idar sensor, It shows the distance of an object in mm. We can collect these points and make a point cloud and make a $3 \mathrm{~d}$ map of the surrounding

Current technologies use radio frequency for controlling the robot which has limited range. Our robot uses the internet as a mode of control, hence it can be controlled from any part of the world. Our rescue robot uses Lidar for 3D mapping which is the future technology being used from smart phones to smart cars. And due to sudden evolution in the technology of $5 \mathrm{~g}$ our rescue robot controlling and communication speed would increase rapidly. Hence has time goes and technology advances in the internet the robot gets evolved and its speed communication speed increases. Current Rescue solutions either use UAV or UGV for rescue operation but our robot comes up with a combination of both UAV and UGV which might bring changes in future designs of the rescue robots.

\section{References}

[1] H. G. Tanner, "Switched UAV-UGV cooperation scheme for target detection," IEEE International Conference on Robotics and Automation, 2007, pp. 3457-3462.

[2] E. Z. MacArthur, D. Carl and C. Crane, "Use of cooperative unmanned air and ground vehicles for detection and disposal of mines," Proceedings of SPIE-The International Society for Optical Engineering, 2005, Vol. 5999, pp. 94-101.

[3] K. DonaldE, E. Z. MacArthur, D. Carl and C. Crane "Unmanned Ground Vehicle state estimation using an Unmanned Air Vehicle," Proceedings of the IEEE International Symposium on Computational Intelligence in Robotics and Automation 2007, pp.473-478.

[4] W. Burgard, D. Fox, M. Moors, R. Simmons, and S. Thrun. "Collaborative multi-robot exploration," In Proceedings of the IEEE International Conference on Robotics and Automation, 2000, pp.1340-1345.

[5] R. G. Simmons , D. Apfelbaum , W. Burgard , D. Fox , M. Moors, S. Thrun , H. L. S. Younes, "Coordination for multi-robot exploration and mapping," Proceedings of the Seventeenth National Conference on Artificial Intelligence and Twelfth Conference on Innovative Applications of Artificial Intelligence, 2000, pp.852-858.

[6] Y. Yang, A. Minai, M. Polycarpou, "Evidential map-building approaches for multi-UAV cooperative search," in Proceedings of the American Control Conference, 2005, pp. 116-121.

[7] D.. W. Casbeer, D. B. Kingston, R. W. Beard, T. W. McLain, S. M. Li, and R. Mehra, "Cooperative forest fire surveillance using a team of small unmanned air vehicles," International Journal of Systems Sciences, 2006, vol. 37, no. 6, pp. 351- 360.

[8] H. H. Gonzalez-Banos, J. Latombe, "Navigation strategies for exploring indoor environments," Journal of Robotic Systems, 2002, 21(10-11):pp.829-848.

[9] M.T. Bryson and S. Sukkarieh, "Co-operative localisation and mapping for multiple UAVs in unknown environments," IEEE/AIAA Aerospace Conference, 2007, pp.1-12.

[10] K. Fregene, D. Kennedy, R. Madhavan, L. E. Parker, D. W. L. Wang, "A class of intelligent agents for coordinated control of outdoor terrain mapping UGVs," Eng. Appl. of AI 18(5), 2005, pp.513-531.

[11] Nikolas Giakoumidis, Jin U Bak, Javier V. Gómez, Nikolaos Mavridis, Arber Llenga Pilot-scale development of a UAV-UGV hybrid with air-based UGV path planningConference Paper · December 2012 DOI: 10.1109/FIT.2012.43

[12] R. Volpe, T. Ohm, R. Petras, R. Welch, J. Balaram, and R. Ivlev, “A prototype Manipulation System for Mars Rover Science Operations", in Proc. of IEEE/RSJ Int. Conf. on intelligent Robots and Systems, Grenoble France, pp. 1486-1492 (1997). 
[13] Dr. Punith Kumar M B, D Manikant Amaresh Savadatti "Virobot the Artificial Assistant Nurse for Health Monitoring, Telemedicine and Sterilization through the Internet" International Journal of Wireless and Microwave Technologies, pp. , 1626

[14] K. Kato, S. Hirose, "Development of Quardruped Walking Robot, TITAN-IX -Mechanical Design Concept and Application for the Humanitarian Demining Robot", Advanced Robotics, 15, 191-204 (2001)

[15] Md. Moniruzzaman, Md. Saniat Rahman Zishan, Sabbir Rahman, Sanwar Mahmud, Antar Shaha,"Design and Implementation of Urban Search and Rescue Robot", International Journal of Engineering and Manufacturing(IJEM), Vol.8, No.2, pp.12-20, 2018.DOI: $10.5815 / \mathrm{ijem} .2018 .02 .02$

\section{Authors' Profiles}

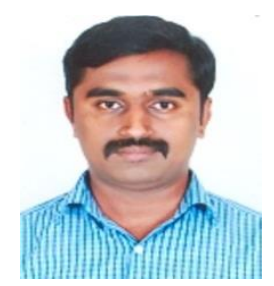

Dr. Punith Kumar M B was born in Mandya, India, in 1985. He received the B.E. Degree in Electronics and Communication Engineering from The National Institute of Engineering, Mysore in 2007, and the M.Tech in VLSI Design and Embedded Systems from PES College of Engineering, Mandya under the The Visvesvaraya Technological University (VTU), Belgaum in 2010 and Ph.D. degrees in Electronics from the University of Mysore (UoM), Mysore, India, in 2017.

In 2007, he joined the Department of Electronics and Communication Engineering as a Lecturer in JVIT, Bidadi, Bangalore. In 2010, he joined the Department of Electronics and Communication Engineering as a Assistant Professor in BGS Institute of Technology, BG Nagar, Mandya. In 2017, he joined the Department of Electronics and Communication Engineering as a Associate Professor in PES College of Engineering Mandya. His current research interests include image processing, video processing, video shot detection etc. Dr Punith Kumar M B is a Life Member of the Indian Society for Technical Education (ISTE) and Associate Member of the Institution of Engineers (AMIE), Member of IEEE. He was the Judge, Chairperson and Review member for the National and International Conference.

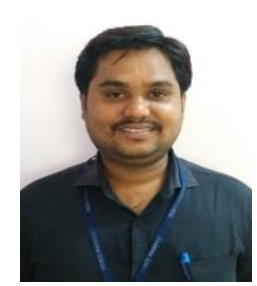

Mr. Sumanth S was born in Mandya, India, in 1989. He received the B.E. Degree in Electronics and Communication Engineering from Rajarajeswari College of Engineering, Bengaluru in 2011, and the M.Tech in VLSI Design and Embedded Systems from PES College of Engineering, Mandya under the The Visvesvaraya Technological University (VTU), Belagavi in 2013.

In 2014, he joined the Department of Electronics and Communication Engineering as an Assistant Professor in PES College of Engineering Mandya. His current research interests are image processing, video processing, Robotics.

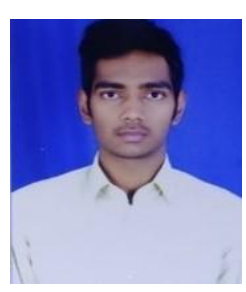

Manikant Amaresh Savadatti student of the Dept, of ECE, PESCE, Mandya, India. He is interested in carryout the projects based on the image processing and Robot, IOT.

How to cite this paper: Punith Kumar M B, Sumanth S, Manikant Amaresh Savadatti, " Internet Rescue Robots for Disaster Management", International Journal of Wireless and Microwave Technologies(IJWMT), Vol.11, No.2, pp. 13-23, 2021.DOI: 10.5815/ijwmt.2021.02.02 